\title{
Congenital Morgagni hernia in a middle-aged woman presenting with chest pain
}

\author{
Hernia congénita de Morgagni en una mujer de mediana edad que presenta dolor torácico \\ Naser Khezerlou', Kamran Mohammadi ${ }^{1}$, Leili Pourafkari², and Nader D. Nader ${ }^{3 *}$ \\ ${ }^{1}$ Cardiovascular Research Center, Tabriz University of Medical Sciences. Tabriz, Iran; ${ }^{2}$ Catholic Health System, University at Buffalo; ${ }^{3} \mathrm{Jacobs}$ School \\ of Medicine and Biomedical Sciences, University at Buffalo, Buffalo, NY, USA
}

A 56-year-old woman with a history of type II diabetes, hypertension, and hyperlipidemia presented to the emergency department with retrosternal chest pain that had increased over the past 5 days. She was experiencing a localized chest pressure that was aggravated with eating and physical activity. The medical history was negative for coronary artery disease or any other cardiac diseases. On electrocardiogram, normal sinus rhythm was detected with normal appearing ST-T segments. Cardiac troponin I was below the detectable

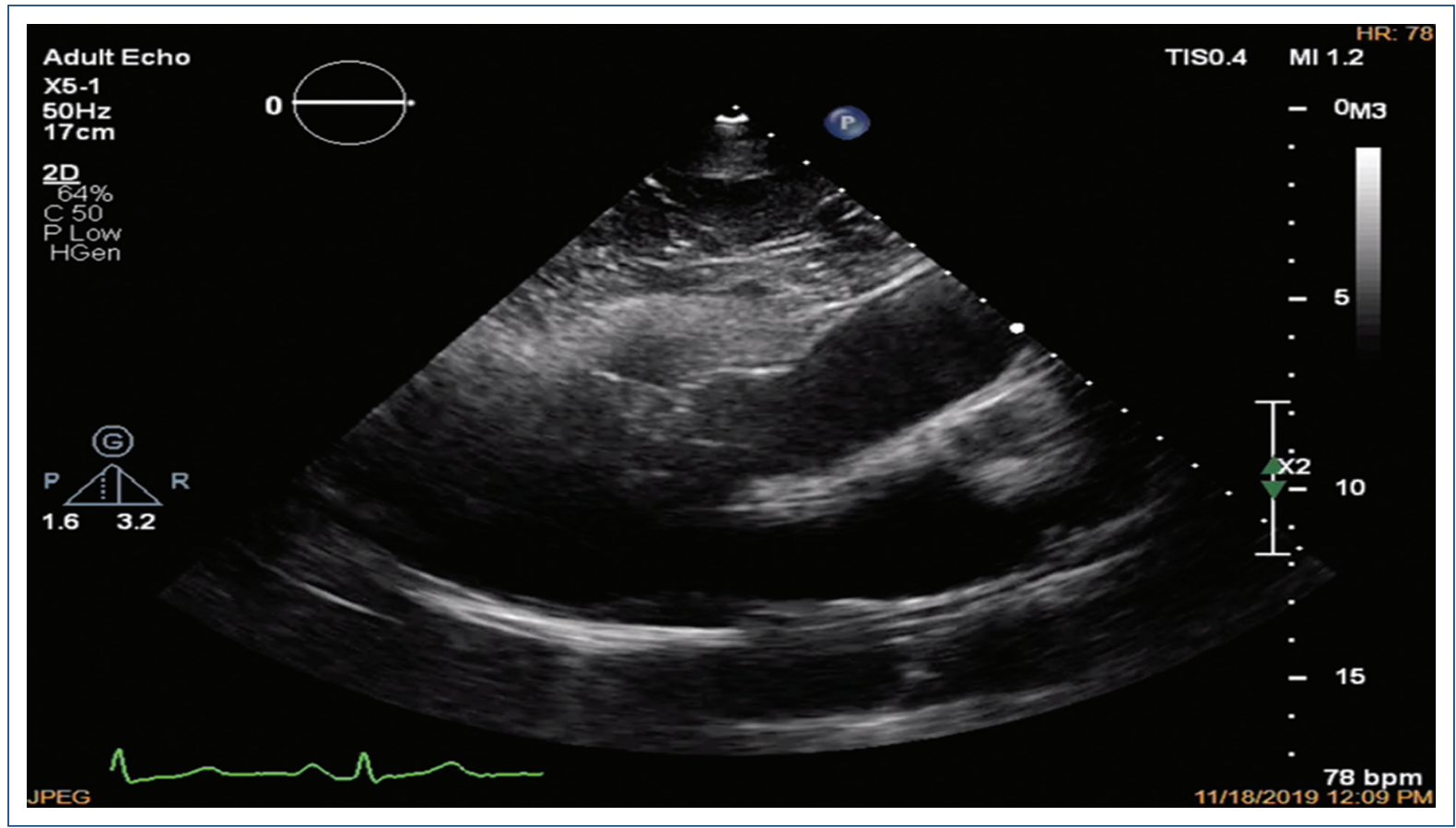

Figure 1. Long-axis parasternal echocardiogram showing retrosternal mass. Arrow indicates pericardium and asterisk indicates hernia contents.

Correspondence:

*Nader D. Nader

E-mail: nadernd@gmail.com
Date of reception: 30-04-2020

Date of acceptance 18-06-2020 DOI: 10.24875/ACM.20000191
Available online: 12-11-2020 Arch Cardiol Mex (Eng). 2021;91(2):246-248

www.archivoscardiologia.com

1405-9940 / @ 2020 Instituto Nacional de Cardiología Ignacio Chávez. Published by Permanyer. This is an open access article under the CC BY-NC-ND license (http://creativecommons.org/licenses/by-nc-nd/4.0/). 


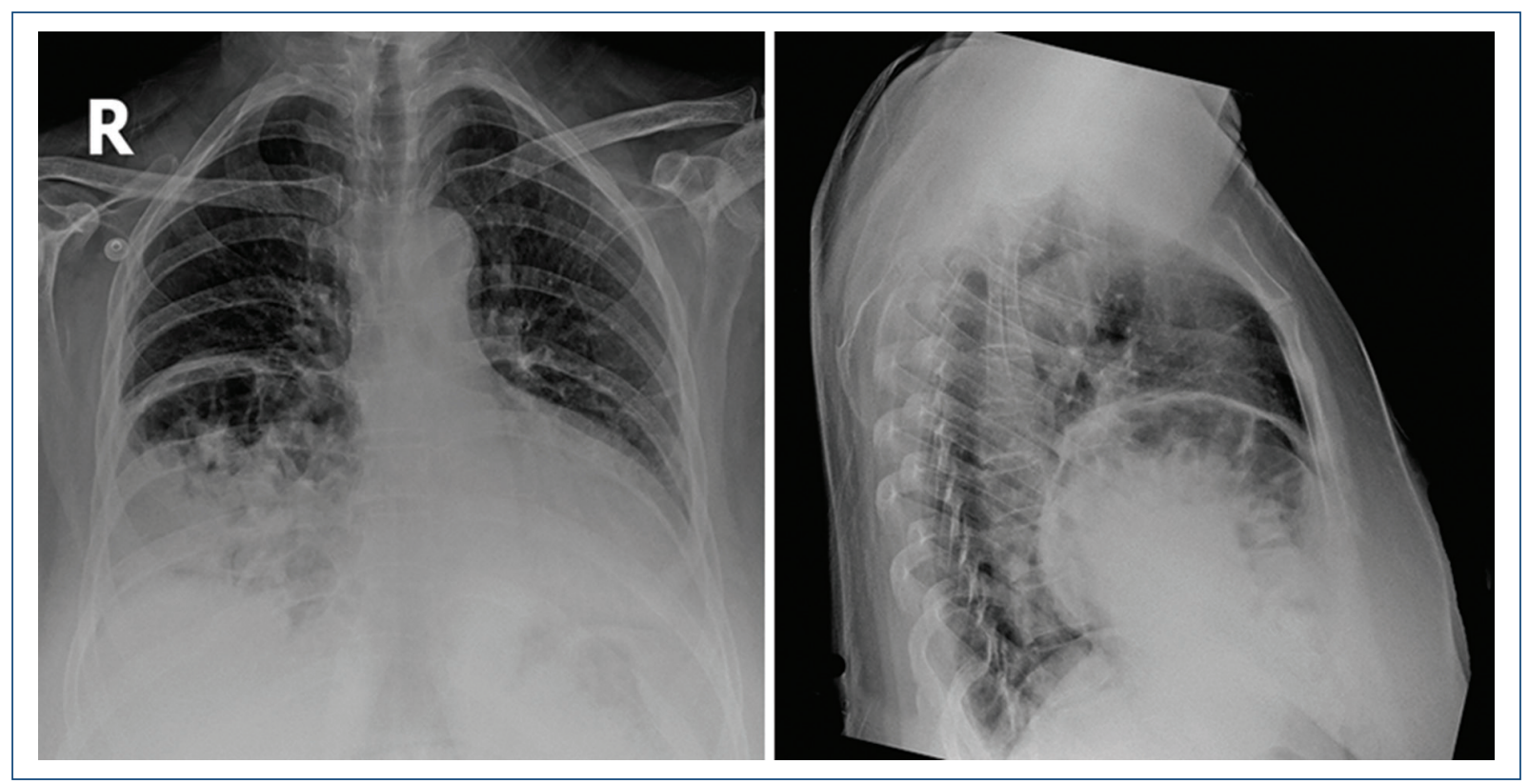

Figure 2. PA and lateral chest X-ray showing gas-containing opacity in the right and anterior side of thorax with mediastinal shift to the left suggesting diaphragmatic hernia.
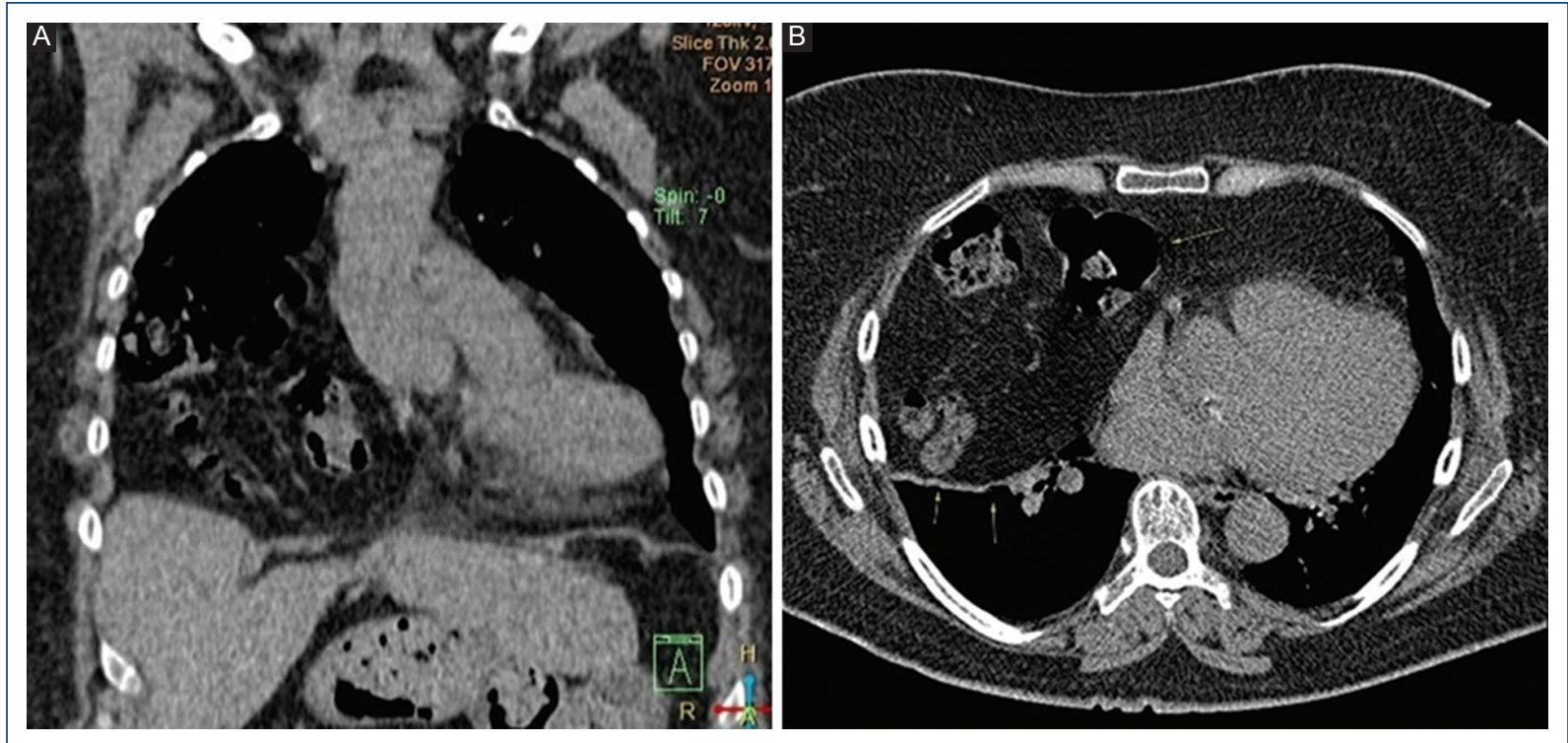

Figure 3. A: coronal and axial B: chest computed tomography views showing large Morgagni hernia with protrusion of omental fat and bowel contents in anterior and mostly right side of thoracic cavity.

level. Transthoracic echocardiography (TTE) showed normal left ventricular systolic function with moderate left ventricular hypertrophy, Grade I diastolic dysfunction, and no regional wall motion abnormalities. There was no significant valvular dysfunction. However, a retrosternal mass anterior to the right ventricle was visualized (Fig. 1). Posterior-anterior and lateral chest radiographs were obtained, which showed gas-containing opacity in the right and anterior side of thorax with mediastinal shift to the left suggesting diaphragmatic hernia (Fig. 2). Chest computed tomography showed large Morgagni hernia with protrusion of omental fat and bowel contents to anterior and right side of thoracic cavity (Fig. 3). Thoracic surgery was consulted and the patient 
was scheduled for surgical repair of the diaphragmatic defect.

Morgagni hernias are rarely encountered in adult population and account for approximately $3 \%$ of congenital diaphragmatic hernias. They occur as a result of defect of anterior and typically right side of diaphragm. Omental fat and transverse colon are most commonly herniated structures. These defects are usually discovered during prenatal ultrasound or present with respiratory symptoms early in life ${ }^{1}$. As adult patients are generally asymptomatic or present with vague symptoms, the diagnosis is generally delayed. Although they can be incidentally discovered during an imaging study of the chest, TTE is exceedingly rare method described in visualization of Morgagni hernia $^{2}$. Once diagnosed, surgical repair is generally warranted to prevent complications and strangulation.

\section{Conflicts of interest}

None.

\section{Ethical disclosures}

Protection of human and animal subjects. The authors declare that no experiments were performed on humans or animals for this study.

Confidentiality of data. The authors declare that they have followed the protocols of their work center on the publication of patient data.

Right to privacy and informed consent. The authors have obtained the written informed consent of the patients or subjects mentioned in the article. The corresponding author is in possession of this document.

\section{References}

1. Mohamed M, Al-Hillan A, Shah J, Zurkovsky E, Asif A, Hossain M. Symptomatic congenital morgagni hernia presenting as a chest pain: a case report. J Med Case Rep. 2020;14:13.

2. Kim SH, Kim MG, Kim SJ, Moon J, Kang WC, Shin MS, et al. Unusual diaphragmatic hernias mimicking cardiac masses. J Cardiovasc Ultrasound. 2015;23:107-12. 